\title{
Modeling responses of Daphnia magna to pesticide pulse exposure under varying food conditions: intrinsic versus apparent sensitivity
}

\author{
Barry J. Pieters ${ }^{1,2}, 4$, Tjalling Jager ${ }^{3}$, Michiel H. S. Kraak ${ }^{2}$ and \\ Wim Admiraal ${ }^{2}$
}

(1) Department of System Ecotoxicology, Centre for Environmental Research (UFZ), Permoserstrasse 15, 04318 Leipzig, Germany

(2) Department of Aquatic Ecology and Ecotoxicology, Faculty of Science, University of Amsterdam, Kruislaan 320, 1098 SM Amsterdam, The Netherlands

(3) Department of Theoretical Biology, Vrije Universiteit, de Boelelaan 1085, 1081 HV Amsterdam, The Netherlands

(4) Present address: Laboratory for Ecological Risk Assessment (LER), National Institute for Public Health and the environment (RIVM), P.O. Box 1, Antonie van Leeuwenhoeklaan 9, 3720 BA Amsterdam, The Netherlands

\author{
Barry J. Pieters \\ Email: barry.pieters@rivm.nl \\ Phone: +31-30-2743069 \\ Fax: $+31-30-2744413$
}

Received: 22 April 2006 Accepted: 1 September 2006 Published online: 6 October 2006

\begin{abstract}
Recent studies showed that limiting food conditions resulted in either increased or decreased sensitivity of Daphnia magna to toxicants. It remained unclear whether these contrasting food-dependent alterations in toxicity resulted from differences in intrinsic sensitivity of the daphnids or from changes in toxicokinetics and resource allocation. It is hypothesized here that, if food level only affects accumulation kinetics and resource allocation, then the intrinsic sensitivity to this toxicant should be the same for all food regimes. This hypothesis was investigated using the DEBtox model, which is based on the theory of Dynamic Energy Budgets. We examined results of two recently conducted life-cycle studies on the combined effects of food level and a pulsed exposure to the pyrethroid insecticide fenvalerate (FV) on D. magna. The model described the effects of the time-varying exposure well, and indicated that when the animals did not die from exposure to FV, full reversibility of toxic effects was possible, allowing a complete recovery. Results revealed furthermore that the data from both studies could be described by the same NECs for survival and assimilation, killing rate and tolerance concentration (132 (49.2$228) \times 10^{-6} \mu \mathrm{g} / \mathrm{L}, 0\left(0-1.18 \times 10^{-5}\right) \mu \mathrm{g} / \mathrm{L}, 74.4(55.6-96.4) \mathrm{L}(\mu \mathrm{g} \mathrm{d})^{-1}$ and 5.39 $(2.72-18.5) \times 10^{-3} \mu \mathrm{g} / \mathrm{L}$, respectively). It is therefore concluded that fooddependent FV toxicity can be explained by altered toxicokinetics and resource allocation, but not by changes in the intrinsic sensitivity of the daphnids. This study implies that the effect of pesticide application in the field depends on the trophic state of the receiving water body, but also that full recovery of survivors is possible after FV application.
\end{abstract}

Keywords Effects assessment - DEBtox model - Pesticides - Food limitation Time-varying exposure 
This manuscript describes original research that has not been published elsewhere. All persons listed as authors have participated in the research insofar as to take public responsibility for the content.

\section{Introduction}

Food shortage affects the physiological condition of organisms and may therefore increase their sensitivity to toxicants (Heugens et al. 2001). Many studies have indeed reported an increased sensitivity to a wide range of pollutants of invertebrates grown under limiting food conditions (Chandini 1989; Barry et al. 1995; Enserink et al. 1995; Klüttgen et al. 1996; Rose et al. 2002). However, in the common test species Daphnia, low-food supply causes a reproductive tradeoff resulting in fewer, but larger sized offspring (Glazier 1992; Gliwicz and Guisande 1992), which were found to have a lower sensitivity to toxicants (Baird et al. 1989; Enserink et al. 1990). Thus limiting (maternal) food conditions resulted in both increased and decreased sensitivity to toxicants. In all these studies however, increased or decreased sensitivity was assessed as a change in a crude summary statistic such as the LC50 or EC50 at a certain time point. However, a change in EC50 does not necessarily reflect an effect on the 'intrinsic sensitivity' of the test organism [in this paper, we define intrinsic sensitivity as the relationship between the concentration at the target receptor (e.g., tissue concentrations) and the physiological process that is affected (e.g., assimilation of energy from food)]. At the same intrinsic sensitivity, apparent sensitivity to organic chemicals may differ with food level, because of (1) differences in bioavailability, causing the chemical to reach the target to a lesser extent (Barry et al. 1995), (2) alterations in toxicokinetics, because food affects growth and thereby the uptake and elimination kinetics (Day and Kaushik 1987a), and (3) physiological interactions. This last factor requires some elaboration. At low-food levels, maintenance takes up a larger proportion of the total energy budget, because unlike growth and reproduction, maintenance costs cannot be simply decreased by the organism. As a result, the same percentage change in feeding rate or maintenance costs due to a toxicant will lead to a larger effect on growth and reproduction at low-food levels (Kooijman and Metz 1984). Few studies have actually tested these explanations and the aim of the present study was therefore to analyze whether the contrasting results for the effect of food on sensitivity of daphnids to toxicants were actually the result of a changed intrinsic sensitivity of the test organisms. For this purpose, we examined results of two recently conducted life-cycle studies on Daphnia magna testing the combined effects of food level and a pulsed exposure to the pyrethroid insecticide fenvalerate (FV) (Pieters et al. 2005; Pieters and Liess 2006). Episodic exposure to FV was chosen because a single toxicant dose is especially relevant in agriculture, where pesticide application may result in short-term pulse exposures of aquatic biota due to spray drift, drain flow, or field runoff (Liess et al. 1999). In spite of its relevance, episodic pollution received relatively little attention (McCahon et al. 1991; McCahon and Pascoe 1991; Handy 1994; Naddy et al. 2000; Reinert et al. 2002). Results of our first study (Pieters et al. 2005) showed that limiting postnatal food conditions exacerbated the effects of a $24 \mathrm{~h}$ FV pulse exposure. In contrast, Pieters and Liess (2006) demonstrated that daphnids originating from low-fed mothers were less sensitive to the pulsed FV exposure compared to daphnids born from high-fed mothers (Pieters and Liess 2006). It is hypothesized here that food level only affects accumulation kinetics of FV and resource allocation, but that the intrinsic sensitivity to FV was the same for all food regimes. This hypothesis was investigated using the DEBtox model (see Kooijman and Bedaux 1996), which is based on the theory of Dynamic Energy Budgets (DEB, see Kooijman 2000). Because the model explicitly considers toxicokinetics (and the effects of body size on toxicokinetics) and resource allocation, it was possible to incorporate time-varying FV concentrations and to distinguish between 
effects of food level on the uptake and resource allocation on the one hand, and changes in intrinsic sensitivity of the organism to FV on the other. The applicability of DEBtox to time-varying concentrations was shown earlier by Péry et al. (2001) for effects on survival only.

\section{Materials and methods Experimental data set}

Life-cycle toxicity data from two previous laboratory studies with $D$. magna (Pieters et al. 2005; Pieters and Liess 2006) were used for this study. In short, 21-day Daphnia reproduction tests (OECD 1997) were conducted in which newly released neonates ( $<24 \mathrm{~h}$ old) from a laboratory culture were individually transferred into glass beakers containing $80 \mathrm{~mL}$ of test medium and pulse exposed for $24 \mathrm{~h}$ to several concentrations of FV (control, $0.03,0.1,0.3,0.6,1.0$, 3.2 , and $10.0 \mu \mathrm{g} / \mathrm{L}$ ). After the exposure, offspring were transferred to uncontaminated medium and life-history traits (survival, growth, reproduction) were subsequently monitored during 21 days. Survival and the number of living neonates produced were recorded on a daily basis, and the body lengths of neonates were measured at birth and on days 7, 14, and 21. Daphnids were fed with a suspension of the green algae Desmodesmus subspicatus. The first study was initiated with neonates originating from maternal cohorts cultured at a highfood level (equivalent daily ration: $5.3 \times 10^{5}$ cells $/ \mathrm{mL} ; 0.07 \mathrm{mg}$ carbon/daphnid/day). Next, neonates were exposed to FV at an identical highfood regime in the 'high-food' treatment $(n=15)$, while neonates in the 'lowfood' treatment $(n=20)$ received a low-food level $\left(1.5 \times 10^{5}\right.$ cells $/ \mathrm{mL} ; 0.02 \mathrm{mg}$ carbon/daphnid/day). The second study was initiated with neonates $(n=25)$ originating from maternal cohorts fed with the respective low-food regime (i.e., 'maternal low-food' treatment) and were also exposed to FV at this low-food level. The experimental data set was subjected to the DEBtox model to evaluate whether potential changes in the intrinsic sensitivity of the daphnids and/or changes in FV toxicokinetics determined the influence of food level on FV toxicity. A full description of the model is given in Kooijman and Bedaux (1996), but here we will address the relevant assumptions, equations, and extensions (for variables see also Table 1 ). 
Table 1 Parameter estimates of the high-food $(H F)$, low-food $(L F)$, and the maternal low-food (MLF) experiment, with likelihood-based $95 \%$ confidence intervals given between brackets

\begin{tabular}{|c|c|c|c|c|}
\hline Description & Symbol & Unit & HF and LF simultaneously & MLF separately \\
\hline \multicolumn{5}{|l|}{ Physiological parameters } \\
\hline $\begin{array}{l}\text { Von Bertalanffy growth } \\
\text { rate }\end{array}$ & $r_{\text {в }}$ & $d^{-1}$ & $0.138(0.132-0.143)$ & $\begin{array}{l}0.243(0.238- \\
0.256)\end{array}$ \\
\hline Initial length & $L_{0}$ & $\mathrm{~mm}$ & 0.753 (n.e.) & 0.940 (n.e.) \\
\hline $\begin{array}{l}\text { Length at first } \\
\text { reproduction }\end{array}$ & $L_{\mathrm{p}}$ & $\mathrm{mm}$ & $2.38(2.32-2.42)$ & $2.84(2.79-2.87)$ \\
\hline Maximum length & $L_{\mathrm{m}}$ & $\mathrm{mm}$ & $4.15(4.12-4.18)$ & $4.17(4.14-4.20)$ \\
\hline $\begin{array}{l}\text { Maximum reproduction } \\
\text { rate }\end{array}$ & $R_{m}$ & juv/d & $7.24(6.90-7.53)$ & $17.3(16.3-18.6)$ \\
\hline Scaled food density & $F$ & - & $\begin{array}{l}\text { HF: } 1.0 \text { (n.e.), LF: } 0.818 \text { (0.809- } \\
0.825)\end{array}$ & a \\
\hline \multicolumn{5}{|l|}{ Toxicological parameters } \\
\hline Elimination rate & $k_{e}$ & $d^{-1}$ & $2.37(1.33-10.1) \times 10^{-3}$ & a \\
\hline Blank hazard rate & $h_{0}$ & $d^{-1}$ & $0.665(0.231-2.66) \times 10^{-3}$ & $a$ \\
\hline NEC for survival & $C_{0 s}$ & $\mu g / L$ & $132(49.2-228) \times 10^{-6}$ & $a$ \\
\hline NEC for assimilation & $C_{0 a}$ & $\mu \mathrm{g} / \mathrm{L}$ & $0\left(0-1.18 \times 10^{-5}\right)$ & a \\
\hline Tolerance concentration & $C_{T}$ & $\mu \mathrm{g} / \mathrm{L}$ & $5.39(2.72-18.5) \times 10^{-3}$ & a \\
\hline Killing rate & $b$ & $(\mu g d)^{-1}$ & $74.4(55.6-96.4)$ & a \\
\hline $\begin{array}{l}\text { Maximum water } \\
\text { solubility }\end{array}$ & - & $\mu g / L$ & $0.524(0.473-0.593)$ & a \\
\hline
\end{tabular}

Mode of action is a decrease in assimilation due to fenvalerate

${ }^{a}$ Value from the high- and low-food experiment was used. N.e. is not estimated

\section{Toxicokinetics}

Conform the concept of critical body residues (see McCarthy and Mackay 1993), DEBtox describes that a toxicant is taken up from the environment by an organism before it can elicit an effect. The internal concentration determines the organism's probability to die if this concentration exceeds a certain threshold level. In the present study, the internal concentration of FV was deduced from its concentration in the solution (i.e., external concentration) according to a onecompartment model for kinetics (see Heugens et al. 2003). Since measured internal concentrations were not available, scaled internal concentrations $c_{v}$ (i.e., internal concentrations divided by the bioconcentration factor; BCF) were used. This implies that $c_{v}$ is proportional to the true internal concentration, but with the dimensions of an external concentration $\left(c_{d}\right)$. The body residues are expected to follow one-compartment kinetics, in which the effects of growth are accounted for:

$\frac{\mathrm{d}}{\mathrm{d} t} c_{\mathrm{v}}=\frac{k_{\mathrm{s}}}{l}\left(c \mathrm{~d}-c_{\mathrm{v}}\right)-c \mathrm{v} \frac{\mathrm{d}}{\mathrm{d} t} \ln l^{3}$.

This equation has only two unknowns, the elimination rate $\left(k_{\mathrm{e}}\right)$ and the scaled body length $(I)$, i.e., length as a fraction of the maximum length at abundant food, which occurs twice in this equation. First it is used in the term $k \mathrm{e} / l_{\text {, }}$ reflecting that the rate of uptake and elimination depends on the surface-tovolume ratio of the organism (a large organism takes more time to reach steady state than a small one). The second term in this equation represents dilution by growth. 
The DEBtox software protocol was modified to introduce a time-varying external concentration in Eq. 1. Measurements of actual concentrations were available at the start of the pulse exposure, and for the high-food treatment after 1 and $24 \mathrm{~h}$ (see Pieters et al. 2005). The decrease of actual FV concentration over time was assumed to depend on food level (i.e., algal concentration) due to adsorption of the hydrophobic FV to the algae. Therefore, the declining actual FV concentrations of the low-food experiment were simulated to accommodate sorption to be proportional to the algal density.

\section{Effects on survival}

In DEBtox, the survival probability of individuals is calculated via the hazard rate $\left(h_{c}\right)$. As long as the internal concentration is below a no-effect concentration (NEC), the hazard rate due to toxicant stress is zero; above the NEC, the hazard increases linearly with the concentration above the NEC. Note that the internal concentration and hence the NEC $\left(c_{0 s}\right)$ are both scaled in the dimensions of an external concentration. The hazard rate is given by:

$h_{c}=b\left(c \mathrm{v}-c_{0}\right)_{+}$.

The + indicates that the maximum of the expression between brackets and zero is taken. The proportionality constant $b$ is called the killing rate. It is a measure of the effect of the chemical on survival, when the NEC is exceeded. The hazard rate due to toxicant stress is added to a constant background mortality $h_{0}$ and integrated to yield the survival probability $q$, for any concentration and any time point:

$q(t)=\exp \left[-\int_{0}^{t}\left(h_{c}(\tau)+h_{0}\right) \mathrm{d} \tau\right]$.

\section{Effects on growth and reproduction}

Substances such as FV cause a loss of coordination and immobilization, and therefore result in a reduction of filtration rates. Therefore, the physiological mode of action that is most suited to this case is effects on assimilation. The DEBtox equations below were designed to express this mode of action; a stress factor $s$ is defined depending on the scaled internal concentration $c_{v}$. Equivalent to the hazard rate, the stress factor is zero below the NEC $\left(c_{0 a}\right)$, and above the NEC it is proportional to the internal concentration. The proportionally constant $c_{T}$ has the dimensions of an external concentration, and is therefore called the tolerance concentration for assimilation:

$s=\frac{\left(c \mathrm{v}-c_{a}\right)_{+}}{c_{\mathrm{I}}}$

When no toxicant is present and as long-food density $(f)$ remains constant, growth of the isomorphically growing daphnids reduces in DEB to the Von Bertalanffy growth curve (see Kooijman 2000). A scaled body length is adopted to simplify the equations. Under abundant food levels, $f$ equals 1 , but this value becomes smaller than 1 under limiting food levels. This way, food limitation was included in the model. When the daphnids are exposed to a toxicant affecting assimilation, the scaled body length is given as a function of time and food level by:

$\left.\frac{\mathrm{d}}{\mathrm{d} t} l=r_{\mathrm{B}} \mid f(1-s)-l\right]$.

where $r_{\mathrm{B}}$ is the Von Bertalanffy growth rate constant. Both body length and food density control reproduction. The reproduction rate $(R)$ is given as a satiating 
function of body length (or scaled length $I$ ). As long as the scaled body length is below the scaled length at puberty $\left(I_{\mathrm{p}}\right)$, the reproduction rate is zero. Above the length at puberty, the reproduction rate increases with body size, until it reaches the maximum rate $(R \mathrm{~m})$ when $f=1$. For effects on assimilation, the full equation for the reproduction rate at a constant food level is:

$R=(1-s)^{2} \frac{R_{\mathrm{m}}}{1-l_{\mathrm{p}}^{3}}\left[\frac{g+l}{g+f} f l^{2}-l_{\mathrm{p}}^{3}\right]$.

The dimensionless constant $g$ is the energy investment ratio; the ratio of the volume-specific costs for growth and the fraction of the reserves allocated to growth and maintenance. This parameter generally has little influence on the toxicity parameters, and 1 is a reasonable value for Daphnia (see Kooijman and Bedaux 1996).

\section{Fitting procedure}

The DEBtox model was fitted to the data for all endpoints of the high- and lowfood experiments simultaneously (see Jager et al. 2004). This was done by expressing all model fits in terms of likelihood and combining the separate likelihoods into an overall value that can be maximized. The $95 \%$ confidence intervals were generated using the profile likelihood (see Meeker and Escobar 1995). The full analysis was programmed in MatLab ${ }^{\circledR}$ Version 7.0 (Mathworks, Natick, MA, USA). However, the emerging growth and reproduction parameters for the daphnids could not be incorporated in model runs of the maternal lowfood data set. This was likely the result from the fact that low-fed mothers enhanced their maternal investment per offspring, characterized by producing fewer but larger sized neonates with a different life-history. Therefore, a different set of values for the growth and reproduction parameters was required for the experiment on maternal low food. For this purpose, the maternal low-food data set was analyzed separately, using the toxicological parameters generated by modeling the other two experiments (see Table 1 ).

\section{Results and discussion}

Figure 1 presents the model fits for $D$. magna exposed to different combinations of FV and (maternal) food levels. Table 1 shows the corresponding parameter estimates with their likelihood-based 95\% confidence intervals. 

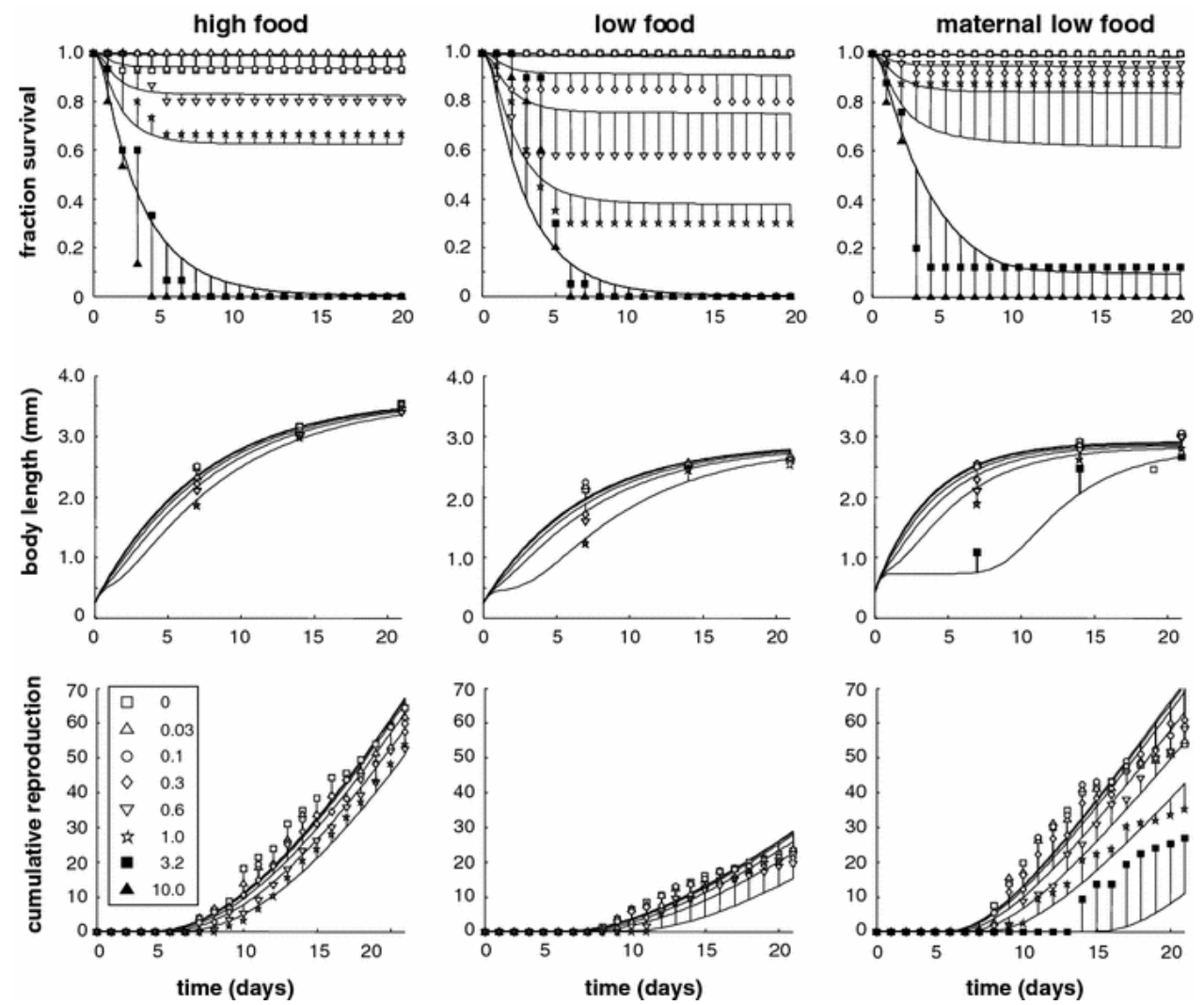

Fig. 1 Model fits of data on survival, body length, and cumulative reproduction of Daphnia magna following a $24 \mathrm{~h}$ pulse exposure to a range of fenvalerate concentrations $(\mu \mathrm{g} / \mathrm{L})$. Graphs show the high- and low-food experiments (fitted simultaneously), and the maternal low-food experiment (fitted separately)

\section{Mode of action of FV}

The growth and reproduction data were well described by the model, assuming a hampered assimilation of food as the mode of action for FV. This assumption corresponds fully with available literature on daphnids and pyrethroids (Day and Kaushik 1987b; Day et al. 1987c; McWilliam and Baird 2002; Christensen et al. 2005; Reynaldi et al. 2006). Due to this effect on assimilation, strong effects were observed on growth curves, which were immediately translated to a delayed onset of reproduction, thereby ultimately affecting the reproduction curves (Fig. 1).

\section{Reversible effects of FV pulse exposure}

Good model fits were obtained for the data, indicating that the model was well able to describe the effects of the time-varying exposure regime, and confirming the link between the estimated internal concentrations and observed effects. Some deviations occurred, especially for survival. However, it must be noted that death is an all-or-nothing effect, and because it is treated as a stochastic process, a good comparison between model and data requires many individuals. Because only a limited number of daphnids survived, one or two more deaths already result in a visual misfit of the model curve. Regarding the deviations for growth and reproduction in the higher exposure treatments, it must be noted that these were likely resulting from the limited number of surviving individuals at high-FV concentrations (the fitting procedure therefore put little weight on these results). $A$ reduced reproduction toward the end of the experiment was observed in all 
treatments, most likely due to variations in food availability caused by the highadult grazing pressure.

The survival data showed that FV-induced mortality still occurred after cessation of exposure which illustrated the slow toxicokinetics of FV, reflected by a low estimate of the elimination rate $k_{\text {e }}$ (Table 1 ). The daphnids obviously accumulated FV to concentrations above the NEC and excreted it so slowly during the post-exposure period that mortality continued in the FV-free period. The higher the exposure concentration, the more FV had been accumulated and hence the longer mortality continued. Due to the combined processes of growth dilution and elimination (see Eq. 1), internal concentrations finally did not exceed the NEC anymore and mortality ceased.

The model assumption that effects relate only to the actual internal concentration implies that if an organism does not die, effects are reversible and full recovery should be possible. The good match between data and model fits indeed confirmed this assumption, as illustrated by the similar reproduction rates between FV treatments and controls in the reproduction curves (see Fig. 1). Similar findings have been reported by Forbes and Cold (2005) who demonstrated that a short-term exposure to the (S)-acid isomer of FV, S-FV, during early larval life of Chironomus riparius had effects on larval survival and developmental rates. However, no lasting effects of $S-F V$ on egg laying or egg viability were observed for surviving individuals.

\section{Sensitivity verus toxicokinetics and resource allocation}

It was hypothesized that, if food level only affects accumulation kinetics and resource allocation, then the intrinsic sensitivity to FV should be the same for all food regimes. This was obviously the case, since all data could be described with the same NECs for survival and assimilation, killing rate, and tolerance concentration (Table 1). Therefore, below we will discuss how food level depending changes in accumulation kinetics and resource allocation may have changed FV toxicity to $D$. magna.

Differing rates of development of the daphnids in the two food regimes with consequent effects on accumulation rates may have contributed to the increased FV toxicity to daphnids in the low-food experiment compared to the high-food experiment. Previous research indicated that the early lifestages of $D$. magna were more sensitive to a short-term FV exposure than adults (Day and Kaushik 1987a), and suggested that this was the result of their smaller body size. Similarly, in the present study the higher surface-to-volume ratio of the slower growing neonates in the low-food experiment compared to the high-food experiment, may have caused a higher bioaccumulation of FV (see Eq. 1). In addition, as daphnids grow during and especially after the $24 \mathrm{~h}$ exposure period, the enhanced toxicity of FV to the daphnids in the low-food experiment may also have been caused by their slower growth dilution of the pesticide. This mechanism is taken into account in the one-compartment kinetics (Eq. 1), and likely decreased internal FV concentrations of the high-fed daphnids much more rapidly over time. Thus, the effect of food level on growth during as well as after pesticide application was likely an important factor modifying FV toxicity.

Differences in resource allocation may also have contributed to the contrasting sensitivities of daphnids between the high- and low-food experiment. The rationale behind this assumption is that $\mathrm{FV}$ effects on assimilation act much stronger under low-food conditions due to a relatively higher demand of energy for somatic maintenance, thereby ultimately affecting growth and reproduction 
stronger than under high-food conditions. This phenomenon has been predicted previously (Jager et al. 2004; Alda Álvarez et al. 2005).

The results of the maternal low-food experiment support the explanation on the interaction of feeding, toxicokinetics and resource allocation given above. $A$ decreased apparent toxicity of FV to daphnids in the maternal low-food experiment agreed with findings of other trans-generational studies using Daphnia exposed to 3, 4-dichloroaniline (Baird et al. 1989) and cadmium (Enserink et al. 1990). Neonate body size at time of birth was considerably larger than in the low-food experiment, decreasing the surface-to-volume dependent accumulation, whereas their growth rate (see Table 1) and hence their growth dilution was much faster. Likewise, the study of Enserink et al. (1990) also suggested a causal relationship between body size and Cd toxicokinetics.

\section{Conclusions}

We demonstrated that the process-based model DEBtox could accurately describe our experimental data, and it could easily account for differing food densities and time-varying exposure concentrations. We proved that effects related only to the actual internal concentration (exceedance of the NEC) and therefore were reversible and full recovery was possible, albeit after some delay, because of the slow toxicokinetics of FV. It is concluded that food-dependent FV toxicity can be explained by altered toxicokinetics and resource allocation, and not necessarily by changes in the intrinsic sensitivity of the daphnids. This implies that the effect of pesticide application on field populations of daphnids will depend on the trophic state of the receiving water body, but also on the reproductive state and size of the animals. Also a full recovery of survivors and resumption of population growth is possible after a FV pulse.

\section{References}

Alda Álvarez O, Jager T, Kooijman SALM, Kammenga JE (2005) Responses to stress of Caenorhabditis elegans populations with different reproductive strategies. Funct Ecol 19:656-664

Baird DJ, Barber I, Bradley M, Calow P, Soares AMVM (1989) The daphnia bioassay: a critique. Hydrobiologia 188/189:403-406

Barry MJ, Logan DC, Ahokas JT, Holdway DA (1995) Effect of algal food concentration on toxicity of two agricultural pesticides to Daphnia carinata. Ecotoxicol Environ Saf 32:273-279

Chandini T (1989) Survival, growth and reproduction of Daphnia carinata (Crustacea: Cladocera) exposed to chronic cadmium stress at different food (Chlorella) levels. Environ Pollut 60:29-45

Christensen BT, Lauridsen TL, Ravn HW (2005) A comparison of feeding efficiency and swimming ability of Daphnia magna exposed to cypermethrin. Aquat Toxicol 73:210-220

Day K, Kaushik NK (1987a) An assesment of the chronic toxicity of the synthetic pyrethroid, Fenvalerate, to Daphnia galeata mendotae, using life tables. Environ Pollut 44:13-26

Day K, Kaushik NK (1987b) Short-term exposure of zooplankton to the synthetic pyrethroid Fenvalerate and its effects on rates of filtration and assimilation of the algae Chlamydomonas reinhardii. Arch Environ Contam Toxicol 16:423-432

Day K, Kaushik NK, Solomon KR (1987c) Impact of Fenvalerate on freshwater enclosed communities filtration rates in zooplankton. Can J Fish Aquat Sci 44:1714-1728 
Enserink L, Luttmer W, Maas-Diepeveen H (1990) Reproductive strategy of Daphnia magna affects the sensitivity of its progeny in acute toxicity tests. Aquat Toxicol 17:15-26

Enserink EL, Kerkhofs MJJ, Baltus CAM, Koeman JH (1995) Influence of food quantity and lead exposure on maturation in Daphnia magna; evidence for a trade-off mechanism. Funct Ecol 9:175185

Forbes VE, Cold A (2005) Effects of the pyrethroid esfenvalerate on life-cycle traits and population dynamics of Chironomus riparius-Importance of exposure scenario. Environ Toxicol Chem 24:78-86

Glazier DS (1992) Effects of food, genotype, and maternal size and age on offspring investment in Daphnia magna. Ecology 73:910-926

Gliwicz ZM, Guisande C (1992) Family planning in Daphnia: resistance to starvation in offspring born to mothers grown at different food levels. Oecologia 91:463-467

Handy RD (1994) Intermittent exposure to aquatic pollutants: assessment, toxicity and sublethal responses in fish and invertebrates. Comp Biochem Physiol C Pharmacol Toxicol Endocrinol 107:171184

Heugens E, Hendriks A, Dekker T, Van Straalen NM, Admiraal W (2001) A review of the effects of multiple stressors on aquatic organisms and analysis of uncertainty factors for use in risk assessment. Crit Rev Toxicol 31:247-284

Heugens EHW, Jager T, Creyghton R, Kraak MHS, Hendriks AJ, Van Straalen NM, Admiraal W (2003) Temperature-dependent effects of cadmium on Daphnia magna: accumulation versus sensitivity. Environ Sci Technol 37:2145-2151

Jager T, Crommentuijn T, Van Gestel CAM, Kooijman SALM (2004) Simultaneous modeling of multiple endpoints in life-cycle toxicity tests. Environ Sci Technol 38:2894-2900

Klüttgen B, Kuntz N, Ratte HT (1996) Combined effects of 3,4-Dichloroaniline and food concentration on life-table data of two related Cladocerans, Daphnia magna and Ceriodaphnia quadrangula. Chemosphere 32:2015-2028

Kooijman SALM, Metz JAJ (1984) On the dynamics of chemically stressed populations: the deduction op population consequences from effects on individuals. Ecotox Environ Saf 8:254-274

Kooijman SALM, Bedaux JJM (1996) Analysis of toxicity tests on Daphnia survival and reproduction. Water Res 30:1711-1723

Kooijman SALM (2000) Dynamic energy and mass budgets in biological systems. Cambridge University Press, Cambridge, UK

Liess M, Schulz R, Liess MHD, Rother B, Kreuzig R (1999) Determination of insecticide contamination in agricultural headwater streams. Water Res 33:239-247

McCarthy LS, Mackay D (1993) Enhancing ecotoxicological modeling and assessment. Body residues and modes of toxic action. Environ Sci Technol 27:1719-1728

McCahon CP, Pascoe D (1991) Brief-exposure of first and forth instar Chironomus riparius larvae to equivalent assumed doses of Cadmium: effects on adult emergence. Water Air Soil Pollut 60:396-403

McCahon CP, Poulton MJ, Thomas PC, Xu Q, Pascoe D, Turner C (1991) Lethal and sub-lethal toxicity of field simulated farm waste episodes to several freshwater invertebrate species. Water Res 25:661671

McWilliam RA, Baird DJ (2002) Postexposure feeding depression: a new toxicity endpoint for use in laboratory studies with Daphnia magna. Environ Toxicol Chem 21:1198-1205 
Meeker WQ, Escobar LA (1995) Teaching about approximate confidence regions based on maximum likelihood estimation. Am Stat 49:48-53

Naddy RB, Johnson KA, Klaine SJ (2000) Response of Daphnia magna to pulsed exposures of Chlorpyrifos. Environ Toxicol Chem 19:423-431

OECD (1997) Guidelines for testing of chemicals: Daphnia magna reproduction test, vol 211. Organization for Economic Cooperation and Development (OECD), Paris, France, pp 1-21

Péry ARR, Bedaux JJM, Zonneveld C, Kooijman SALM (2001) Analysis with time-varying concentrations. Water Res 35:3825-3832

Pieters BJ, Paschke A, Reynaldi S, Kraak MHS, Admiraal W, Liess M (2005) Influence of food limitation on the effects of Fenvalerate pulse exposure on the life history and population growth rate of Daphnia magna. Environ Toxicol Chem 24:2254-2259

Pieters BJ, Liess M (2006) Maternal nutritional state determines the sensitivity of Daphnia magna offspring to short-term Fenvalerate exposure. Aquat Toxicol 76:268-277

Reinert KH, Giddings JM, Judd L (2002) Effects analysis of time-varying or repeated exposures in aquatic ecological risk assessment of agrochemicals. Environ Toxicol Chem 21:1977-1992

Reynaldi S, Duquesne S, Jung K, Liess M (2006) Linking feeding activity and maturation of Daphnia magna following short-term exposure to Fenvalerate. Environ Toxicol Chem 25:1826-1830

Rose RM, Warne MJ, Lim RP (2002) Food concentration affects the life history response of Ceriodaphnia cf. dubia to chemicals with different mechanisms of action. Ecotoxicol Environ Saf $51: 106-114$ 